\title{
TESTICULAR TUMOR PRESENTING AS SCROTAL GANGRENE
}

doi: $10.1590 / \mathbf{S 1 8 0 7 - 5 9 3 2 2 0 0 9 0 0 0 5 0 0 0 1 8}$

\author{
Ernesto Reggio, ${ }^{\mathrm{I}, \mathrm{II}}$ Odival Timm Jr, ${ }^{\mathrm{I}}$ Roberto Gomes Junqueira, ${ }^{\mathrm{I}}$ Marcelo José Sette, ${ }^{\mathrm{I}}$ Jose Bessa Jr.. $\mathrm{III}$
}

\section{INTRODUCTION}

Scrotal gangrene has been described as a necrotizing fascitis and it is most often caused by polymicrobial infections. This paper describes an unusual case of gangrene secondary to an aggressive testicular cancer with an excellent response to chemotherapy.

\section{CASE REPORT}

A 38 year old man with a 14-month history of a large scrotal mass was seen in the emergency room complaining of asthenia, fever, pain, and fetid discharge through the scrotal skin. On physical examination an $11 \mathrm{~cm}$ tumor was revealed in the right hemiscrotum with scrotal gangrene and two ulcers in the left hemiscrotum (Figure 1). The patient had been treated for schizophrenia for 8 years and failed to search for treatment for his scrotal mass. Wide excision of the hemiscrotum including the right testicle was carried out; no other treatment except for antibiotics was carried out. Histopathological examination showed a testicular embryonal carcinoma invading scrotal skin (Figure 2). Consequently, staging was performed. Serum alpha-fetoprotein was $6408 \mathrm{ng} / \mathrm{ml}$, and abdominal CT scan identified a $9 \mathrm{~cm}$ retroperitoneal mass. Chest CT scan was negative for lung metastasis $(\mathrm{TNM}$ stage $=\mathrm{T} 4 \mathrm{~N} 3 \mathrm{M} 0)$. After surgery the patient was referred to the oncology department for chemotherapy and was treated with 3 courses of chemotherapy consisting of bleomycin, etoposide, and platinum. An excellent response to chemotherapy was obtained. The regression rate of retroperitoneal lymph node

I Centro Hospitalar Unimed, Uroclínica de Joinville - Jonville/SC, Brazil. II Pontifícia Universidade Católica do Paraná (PUC PR) - Paraná, Brazil. III Universidade Estadual de Feira de Santana (UEFS) - Feira de Santana/ BA, Brazil.

Email: e reggio@hotmail.com

Tel.: 55473441.9555 metastasis was $95 \%$ on $\mathrm{CT}$, and a $2.1 \mathrm{~cm}$ residual mass in the retroperitoneum has been followed with no evidence of biochemical recurrence after 26 months of follow-up. Due to this response, both the oncologists and the patient's family opted for close surveillance rather than surgery. The scrotal

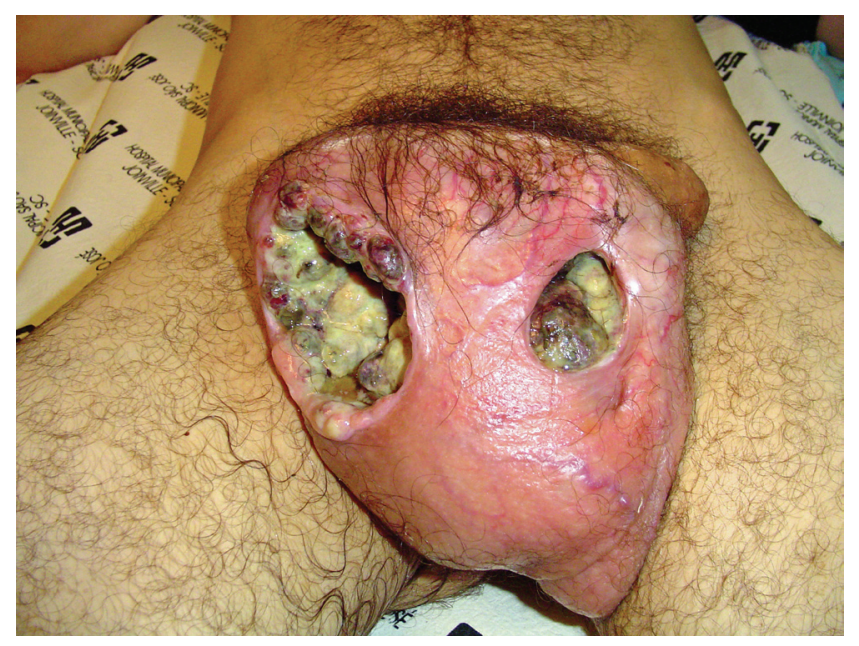

Figure 1 - Extensive necrosis on the right hemiscrotum

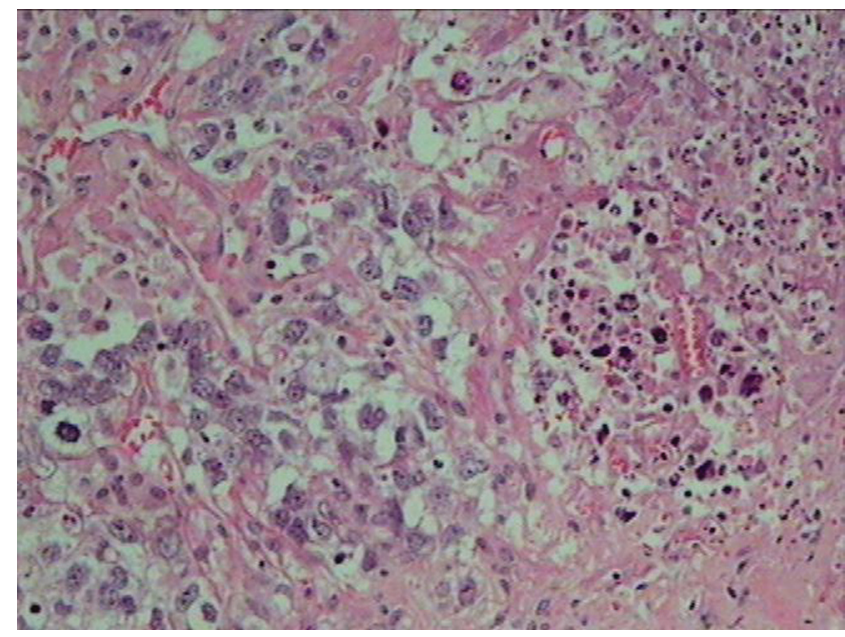

Figure 2 - Histopathological examination 
skin was completely healed with satisfactory functional and cosmetic results and it remains free of local recurrence (Figure 3).

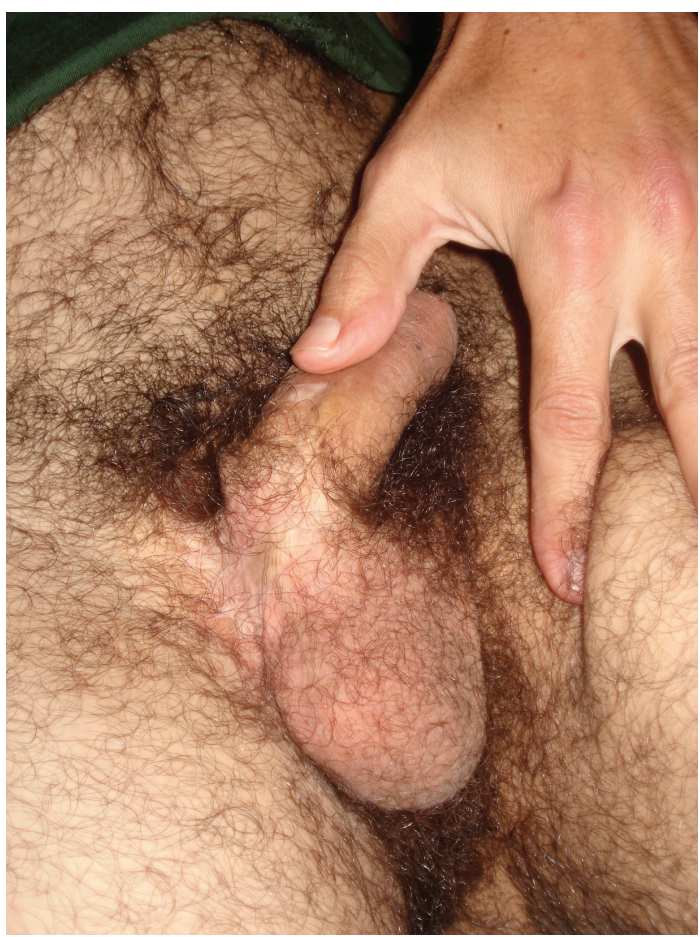

Figure 3 - Complete healing 26 months after excision

\section{DISCUSSION}

Among solid neoplasms, testicular cancer serves as a paradigm for the multimodal treatment and effectiveness of chemotherapy. Since testicular cancer originates in germ cells, which are quite sensitive to radiation and chemotherapy, multidrug regimens provide higher cure rates, decreasing patient mortality. Even advanced stages (such as with the case reported here), are successfully treated with an aggressive combined treatment.

This patient presented with a scrotal necrosis, a severe infectious disease which is life-threatening as it demands prompt and aggressive treatment. Scrotal involvement is a rare condition in testicular cancer since the tunica albuginea is a natural barrier to expansive local growth.

Scrotum is rarely affected by neoplasms. Sanchez-Ortiz et al. ${ }^{1}$ described their experience with scrotal melanoma, an unusual condition associated with a poor prognosis. Softtissue neoplasms can originate in diversified scrotal cells, such as in the epithelioid sarcoma of the scrotum described by Onol et al. ${ }^{2}$ and the granular cell tumor observed by Godoy et al. ${ }^{3}$ However, testicular cancer with extended scrotal involvement and necrosis has been reported by Hyouchi et al. ${ }^{4}$ Due to the giant testicular teratocarcinoma which had invaded scrotum, perineum, and left spermatic cord the patient was treated with three courses of cisplatin, etoposide, and peplomycin. Excellent response to treatment was achieved and the patient was free from recurrence after a short follow-up.

It is interesting to note that no visceral metastasis was detected despite the fact that our patient presented with a local aggressive malignancy. Perhaps this particular oncologic behavior contributed to the success of local and systemic treatment.

An interesting aspect of this case is the delayed diagnosis. Patients with a history of schizophrenia tend to conceal genital diseases or cause them. Rammos et al. ${ }^{5}$ described a recurrent scrotal abscess in a patient who introduced foreign bodies in the scrotum. In our case the patient hid the scrotal enlargement for months; only the strong odor from the scrotal discharges enabled his family to recognize the condition. In spite of the long delay in adequate diagnosis and treatment, chemotherapy provided the patient with full recovery with no evidence of recurrence. The aggressive local behavior and excellent response to the treatment in this case suggests that there may be a wide biological spectrum in germ cell tumors.

\section{CONCLUSION}

High rates of positive response can be achieved with a multimodal treatment for testicular cancer, even at advanced stages.

\section{REFERENCES}

1. Sanchez-Ortiz R, Huang SF, Tamboli P, Prieto VG, Hester G, Pettaway CA. Melanoma of the penis, scrotum and male urethra: a 40-year single institution experience. J Urol. 2005;173:1958-65.

2. Onol FF, Tanidir Y, Kotiloglu E, Bayramicli M, Turhal S, Turkeri LN Proximal type epithelioid sarcoma of the scrotum: a source of diagnostic confusion that needs immediate attention. Eur Urol. 2006;49:406-7.

3. Godoy G, Mufarrij PW, Tsou HC, Torre P, Taneja SS. Granular Cell Tumor of Scrotum: A Rare Tumor of the Male External Genitalia.
Urology. 2008;72:716. e7-9.

4. Hyouchi N, Yamada T, Takeuchi S, Machida T, Kanou H, Tanizawa A, et al. Giant testicular tumor associated with scrotal gangrene: a case report. Hinyokika Kiyo. 1997;43:237-40 .

5. Rammos L, Kapralos V, Gerzelis I, Zagilas D, Karidis G. Multiple recurrent scrotal abscesses and metallic foreign bodies. Prog Urol. 1999;9:541-3. 